\title{
AGR2 ameliorates tumor necrosis factor- $\alpha$-induced epithelial barrier dysfunction via suppression of NF-кB p65-mediated MLCK/p-MLC pathway activation
}

\author{
XIAOLIN YE and MEI SUN \\ Department of Paediatrics, China Medical University Affiliated with Shengjing Hospital, \\ Shenyang, Liaoning 110004, P.R. China
}

Received October 25, 2016; Accepted March 13, 2017

DOI: $10.3892 /$ ijmm.2017.2928

\begin{abstract}
Intestinal epithelial barrier dysfunction plays a critical role in the pathogenesis of inflammatory bowel disease (IBD). Anterior gradient protein 2 homologue (AGR2) assists in maintaining intestinal homeostasis in dextran sulphate sodium-induced mouse ileocolitis; however, it is unclear whether it modulates intestinal barrier function. Our study aimed to investigate the protective role of AGR2 in tumor necrosis factor (TNF)- $\alpha$-induced intestinal epithelial barrier injury. Caco- 2 cell monolayers were pre-transfected with an AGR2 plasmid and then exposed to TNF- $\alpha$. Epithelial permeability was assessed by detecting transepithelial electrical resistance and fluorescein isothiocyanate-dextran $(40 \mathrm{kDa})$ flux. The protein expression levels of zonula occludens-1 (ZO-1), occludin, claudin-1, myosin light chain kinase (MLCK)/p-MLC, and nuclear factor (NF)- $\mathrm{B}$ p 65 were determined by western blotting. In addition, the cellular distributions of ZO-1, occludin, F-actin, and NF- $\kappa$ B p65 were evaluated by immunofluorescence staining. The results showed that the AGR2 mRNA and protein expression levels were both decreased in the Caco-2 cell monolayers, while AGR2 overexpression significantly ameliorated TNF- $\alpha$-induced epithelial barrier hyperpermeability, increased the expression of tight junction (TJ) proteins
\end{abstract}

Correspondence to: Dr Mei Sun, Department of Paediatrics, China Medical University Affiliated with Shengjing Hospital, 36 Sanhao Street, Shenyang, Liaoning 110004, P.R. China E-mail: sunm@sj-hospital.org

Abbreviations: AGR2, anterior gradient protein 2 homologue; IBD, inflammatory bowel disease; TNF- $\alpha$, tumor necrosis factor- $\alpha$; MLCK, myosin light chain kinase; p-MLC, phosporylated myosin light chain; TEER, transepithelial electrical resistance; TJ, tight junction

Key words: anterior gradient protein 2 homologue, inflammatory bowel disease, intestinal epithelial barrier, tumor necrosis factor- $\alpha$, tight junction, myosin light chain kinase and stabilized the cytoskeletal structure. Furthermore, AGR2 inhibited the changes in MLCK, MLC and p-MLC expression in response to TNF- $\alpha$ stimulation. Collectively, our study suggests that AGR2 inhibits TNF- $\alpha$-induced Caco- 2 cell hyperpermeability by regulating TJ and that this protective mechanism may be promoted by inhibition of NF- $\kappa \mathrm{B}$ p65-mediated activation of the MLCK/p-MLC signaling pathway.

\section{Introduction}

Disruption of intestinal epithelial barrier function is a key characteristic of inflammatory bowel disease (IBD) (1-4). An intact intestinal epithelial barrier is essential for defending against the invasion of antigens and for maintaining the internal environment, which separates luminal contents within the body and prevents luminal bacterial and microbial translocation. Intestinal barrier dysfunction leads to entry of intestinal bacteria into the body, causing local or systemic inflammatory responses $(5,6)$. Repair of the intestinal mucosa has become a standard criterion for evaluating the efficacy of IBD treatments (7). Therefore, effective maintenance of intestinal epithelial barrier function is of great importance in IBD.

Integrity of the intestinal epithelial barrier depends on a complex network of intercellular tight junctions (TJs) and cytoskeletal structures, and disruption of this network results in impaired barrier function (8-11). TJ proteins are located at the apical ends of epithelial cells, which contain both transmembrane (e.g., occludin, claudin and junctional adhesion molecules) and cytoplasmic zonula occludens ( $\mathrm{ZO})$ proteins. As the determinant of epithelial permeability, TJ proteins are the most important component of the epithelial mucosal barrier $(12,13)$. Damage to intestinal epithelial TJs leads to increased intercellular permeability, allowing for bacteria and endotoxins to penetrate the mucosa and enter into circulation, resulting in severe inflammatory responses $(14,15)$. Rearrangement and redistribution of cytoskeletal F-actin also leads to changes in intestinal epithelial barrier permeability (16).

Tumor necrosis factor (TNF)- $\alpha$ is a multifunctional proinflammatory cytokine which participates in the pathogenesis of IBD (17). TNF- $\alpha$ is associated with damage to 
intestinal TJs and cytoskeletal rearrangements, which lead to increased intestinal permeability. It also promotes release of the inflammatory factors interleukin (IL)-8, IL-17 and interferon (IFN) $-\gamma(18-20)$. Both the myosin light chain kinase (MLCK)/p-MLC pathway and nuclear factor (NF)- $\mathrm{B}$ activation have key roles in TNF- $\alpha$-mediated damage to the intestinal barrier $(21,22)$. Activation of the MLCK/p-MLC pathway leads to upregulation of the expression of TJ proteins, alterations in cell adhesion and migration, and cytoskeletal rearrangements $(16,23,24)$.

Anterior gradient protein 2 homologue (AGR2) is a member of the protein disulphide isomerase family that plays an important role in maintaining intestinal homeostasis in IBD (25). The prevalence of AGR2 gene mutations is higher in patients with IBD than in the general population (26). In animal models of IBD, AGR2 has been shown to maintain homeostatic functions in intestinal Paneth and goblet cells (27), and AGR2-knockout mice have been reported to be susceptible to the development of severe ileocolitis (28). Thus, AGR2 is essential for maintaining the intestinal epithelial cell function in IBD, but it is unclear whether it regulates intestinal epithelial barrier function.

Therefore, we investigated the roles of AGR2 in epithelial permeability, TJ protein expression, and cytoskeletal structure. Furthermore, we evaluated the mechanism by which AGR2 ameliorates TNF- $\alpha$-mediated damage to the intestinal barrier.

\section{Materials and methods}

Cell culture and preparation. Caco-2 cells were purchased from the Chinese Academy of Sciences (Shanghai, China). The cells were cultured in RPMI-1640 medium containing 10\% fetal bovine serum (FBS; ScienCell Research Laboratories, San Diego, CA, USA) at $37^{\circ} \mathrm{C}$ in $5 \% \mathrm{CO}_{2}$. For the monolayer model, cells were plated at $3 \times 10^{4} / \mathrm{ml}$ onto Transwell filters with a $0.4-\mu \mathrm{m}$ pore size (Millipore, Billerica, MA, USA) and cultured for 21 to 28 days prior to the experiments. The culture medium was changed every 2 days. The experimental monolayers were pre-transfected with an AGR2 plasmid and were then stimulated with $100 \mathrm{ng} / \mathrm{ml}$ rhTNF- $\alpha$ for $48 \mathrm{~h}$.

Plasmid construction and transfection. A pcDNA3.1 eukaryotic expression vector carrying the human AGR2 gene was constructed. Cells were transfected with either a pcDNA3.1-AGR2 plasmid or a pcDNA3.1 vector control plasmid. For transfection, the cells were incubated in $100 \mu 1$ Opti-MEM medium containing $8 \mu \mathrm{l}$ Lipofectamine 2000 (both from Invitrogen Life Technologies, Carlsbad, CA, USA) for $5 \mathrm{~min}$, followed by the addition of $2 \mu \mathrm{g}$ plasmids and incubation for another $20 \mathrm{~min}$. The mixtures were then transferred to 6 -well plates and cultured for $4 \mathrm{~h}$. Subsequently, the transfection medium was substituted with culture medium, and the transfected cells were cultured at $37^{\circ} \mathrm{C}$ in $5 \% \mathrm{CO}_{2}$.

Determination of transepithelial electrical resistance (TEER). Caco-2 cells were seeded onto Transwell chambers, and epithelial permeability was assessed by evaluating TEER using a Millicell ERS-2 Voltohmmeter (Millipore). Two wells containing only culture medium were used as blank controls. Measurements were carried out at a constant temperature at three different locations within each chamber. The mean value of three consecutive measurements was used to calculate TEER.

$\operatorname{TEER}\left(\Omega \cdot \mathrm{cm}^{2}\right)=($ measured TEER - control TEER) $\mathrm{x}$ effec tive membrane area of the cell culture well.

Measurement of paracellular marker fluorescein isothiocyanate (FITC)-dextran 40 (FD-40) (40 kDa) flux. The monolayers were washed with HBSS, and the RPMI-1640 medium in the apical chambers was replaced with FITC-conjugated dextran $(80 \mu \mathrm{g} / \mathrm{ml}, 40 \mathrm{kDa}$; Sigma-Aldrich, St. Louis, MO, USA) in HBSS. The monolayers were incubated at $37^{\circ} \mathrm{C}$ for $1 \mathrm{~h}$. Then, the fluorescence of the culture medium in the lower chambers was detected with a fluorescence plate reader (Varioskan Flash; Thermo Electron Corporation, Vantaa, Finland) at excitation and emission wavelengths of 427 and $536 \mathrm{~nm}$, respectively. Fluorescein concentrations were determined by comparison to a standard curve.

Fluorescence transmittance $(\%)=$ FITC-dextran concentration in the lower chamber/FITC-dextran concentration added to the upper chamber $\mathrm{x} 100$.

Quantitative (real-time) PCR ( $q$ PCR). TRIzol was utilized to extract total RNA from the cells, and reserve transcription was carried out using a PrimeScript RT reagent kit (Takara Bio Inc., Shiga, Japan). qPCR was performed using a SYBR Premix Ex kit (Takara Bio Inc.) and a Bio-Rad iQ5 Real-Time system (Bio-Rad Laboratories, Inc., Hercules, CA, USA). The primers for this study were as follows: $\beta$-actin forward, 5 '-CTT AGTTGCGTTACACCCTTTCTTG-3' and reverse, 5'-CTGTC ACCTTCACCGTTCCAGTTT-3'; AGR2 forward, 5'-GCATT CTTGCTCCTTGTGG-3' and reverse, 5'-GACTGTGTGGGC ACTCATCC-3'. Expression levels were calculated using the $2^{-\Delta \Delta \mathrm{CT}}$ method.

Western blotting. Radioimmunoprecipitation assay (RIPA) and phenylmethanesulfonyl fluoride (PMSF) lysate buffers were used to extract total protein from the Caco- 2 cells. The protein concentrations of the extracted samples were determined by bicinchoninic acid assay. These concentrations were adjusted by addition of distilled water. Equal volumes of sample buffer and boiling water were mixed, and the mixtures were boiled for $5 \mathrm{~min}$ to denature the proteins. Equal amounts of proteins $(40 \mu \mathrm{g})$ for each sample were loaded onto SDS-PAGE gels for electrophoresis, and then transferred to PVDF membranes and blocked by 5\% non-fat milk for $1 \mathrm{~h}$. Next, incubation was carried out with the primary antibodies, including anti-AGR2 (ab76473; 1:3,000), anti-ZO-1 (ab59720; 1:500), anti-claudin-1 (ab15098; 1:500), anti-occludin (ab31721; 1:500) anti-MLCK (ab76092; 1:5,000) and anti-NF-кB (ab207297; 1:500) (all from Abcam, Cambridge, MA, USA), anti-p-MLC (\#3671; 1:750) and anti-MLC (\#8505; 1:750) (both from Cell Signaling Technology, Inc., Danvers, MA, USA) and anti- $\beta$-actin (20536-1-AP; 1:2,000; Proteintech, Wuhan, China) overnight at $4^{\circ} \mathrm{C}$. After that, incubation was carried out with horseradish peroxidase (HRP)-conjugated secondary antibodies (SA00001-2; 1:5,000; Proteintech) for $2 \mathrm{~h}$ and visualized by electrochemiluminescence (ECL). Protein bands were detected using Gel-Pro Analyser 4.0 (Media Cybernetics, Inc., Rockville, MD, USA). 
A

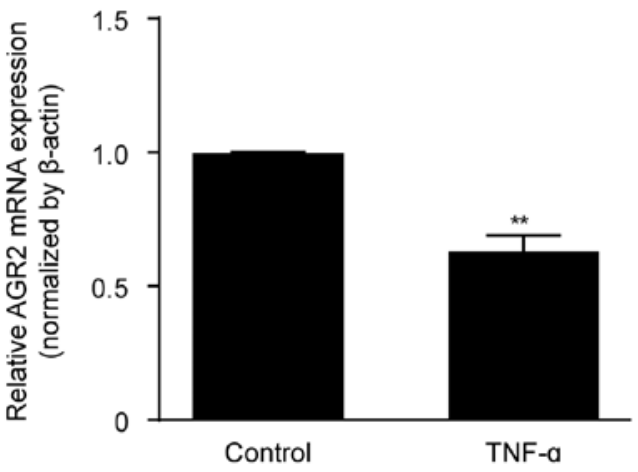

B
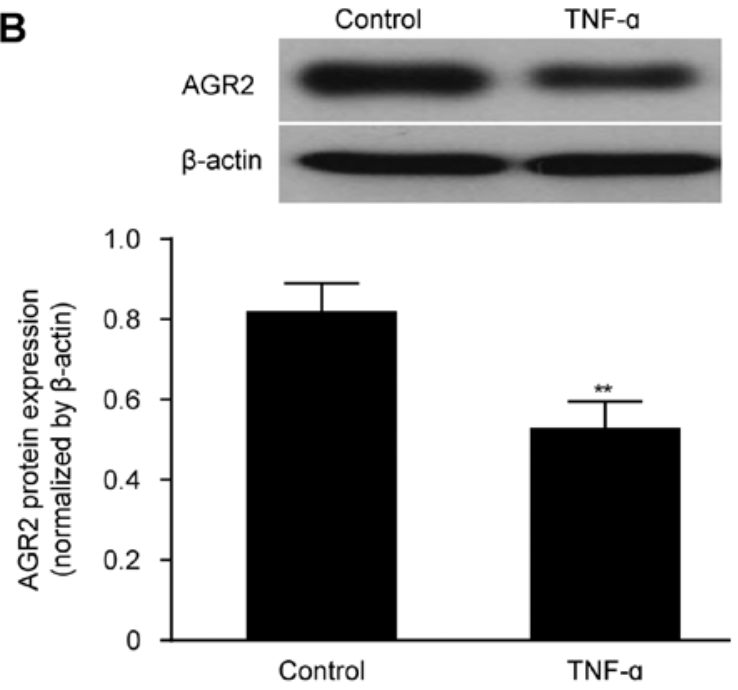

Figure 1. (A) Anterior gradient protein 2 homologue (AGR2) mRNA expression was significantly reduced in the rhTNF- $\alpha$-induced intestinal epithelial barrier injury model. (B) AGR2 protein expression was also decreased by rhTNF- $\alpha$. The results are presented as the mean \pm SD. ${ }^{* *} \mathrm{P}<0.01$ compared with the controls.

Immunofluorescence staining. Caco-2 monolayers were pre-transfected with AGR2 gene plasmids or a control plasmid vector; after $24 \mathrm{~h}, 100 \mathrm{ng} / \mathrm{ml} \mathrm{TNF}-\alpha$ was added. After $48 \mathrm{~h}$ of TNF- $\alpha$ exposure, the cultures were fixed with $4 \%$ polyformaldehyde for $15 \mathrm{~min}$ and then incubated with $0.5 \%$ Triton X-100 for $30 \mathrm{~min}$. Subsequently, the cultures were incubated with goat serum for $15 \mathrm{~min}$. After that, the cells were incubated with primary antibodies including anti-F-actin (ab205; 1:100), anti-ZO-1 (1:200), anti-occludin (1:100) and anti-NF- $\mathrm{B}$ p65 (1:200) (all from Abcam) overnight at $4{ }^{\circ} \mathrm{C}$. Next, they were incubated with a fluorescent-labelled goat anti-rabbit $\operatorname{IgG}(\mathrm{H}+\mathrm{L})$ secondary antibody (SA00003-1; 1:200; Proteintech) for $1 \mathrm{~h}$ at room temperature. After nuclear counterstaining of the cells with 4',6-diamidino-2-phenylindole (DAPI) (Life Technologies, Thermo Fisher Scientific, Inc., Waltham, MA, USA), images were captured by laser scanning fluorescence microscopy (TCS SP5; Leica Microsystems, Wetzlar, Germany) at x400 magnification.

Statistical analysis. Assays were performed at least in triplicate, and the results were reported as the mean \pm SD. The significance of differences was assessed by analysis of variance (ANOVA) or the t-test using SPSS version 21.0 software (SPSS, Inc., Chicago, IL, USA). A P $<0.05$ was considered statistically significant.

\section{Results}

TNF- $\alpha$ decreases AGR2 expression in confluent monolayers of cultured intestinal epithelial cells. Caco-2 cell cultures reached confluence by day 21 , forming monolayers consisting of irregular polygonal cells with a cobblestone appearance and intercellular TJs, as observed by light microscopy. TEER remained stable at $>600 \Omega / \mathrm{cm}^{2}$, indicating the successful establishment of the in vitro intestinal epithelial barrier model. TNF- $\alpha(100 \mathrm{ng} / \mathrm{ml})$ was then added to the model cultures, and after $48 \mathrm{~h}$, the expression level of AGR2 was detected. Both the AGR 2 mRNA and protein expression levels were obvi- ously decreased by TNF- $\alpha$ exposure compared with the levels in the untreated control monolayers (Fig. 1A and B).

Construction of an AGR2 overexpression model by transfection of an AGR2 or control plasmid. AGR2 or control plasmid vectors were transfected in vitro into Caco- 2 cell monolayers, and AGR2 mRNA expression was determined by qRT-PCR after $24 \mathrm{~h}$. After another $48 \mathrm{~h}$ of culturing, AGR2 protein expression was measured by western blotting and compared with that in the controls. The results showed that both the AGR 2 mRNA and protein expression levels were significantly increased in the Caco-2 cells transfected with the AGR2 plasmids (Fig. 2A and B). These findings indicate the successful construction of the AGR2 plasmid.

AGR 2 reduces $T N F$ - $\alpha$-induced increase in permeability of intestinal epithelial cell monolayers. Caco-2 cell monolayers were transfected with AGR2 or control plasmids, and after incubation for $24 \mathrm{~h}, 100 \mathrm{ng} / \mathrm{ml} \mathrm{TNF}-\alpha$ was added. Permeability of the intestinal epithelial cell monolayers was assessed by measuring TEER and FD-40 flux after $48 \mathrm{~h}$ of incubation with TNF- $\alpha$. The results showed that TEER was significantly lower and FD-40 flux was significantly higher in the TNF- $\alpha$ stimulated monolayers compared with that noted in the control monolayers (Fig. 3A and B). AGR2 plasmid transfection significantly inhibited the decrease in TEER as well as the increase in FD-40 flux induced by TNF- $\alpha$ (Fig. 3A and B), indicating that AGR2 ameliorated the TNF- $\alpha$-induced increase in permeability of the model system.

AGR2 inhibits the decreased expression of ZO-1, occludin, and claudin-1 TJ proteins induced by TNF- $\alpha$. Disruption of TJs is an important component of altered intestinal epithelial barrier function (29). We then examined the role of AGR2 and TNF- $\alpha$ in regulating $\mathrm{TJ}$ proteins. Western blotting confirmed that TNF- $\alpha$ stimulation decreased the expression of ZO-1, claudin-1 and occludin (Fig. 4A-C) and that AGR2 plasmid transfection attenuated these decreases in expression (Fig. 4A-C). Immunofluorescence staining showed that in the control group, 
A

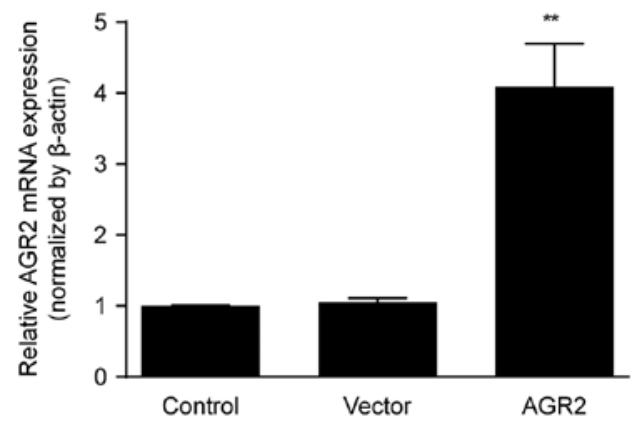

B
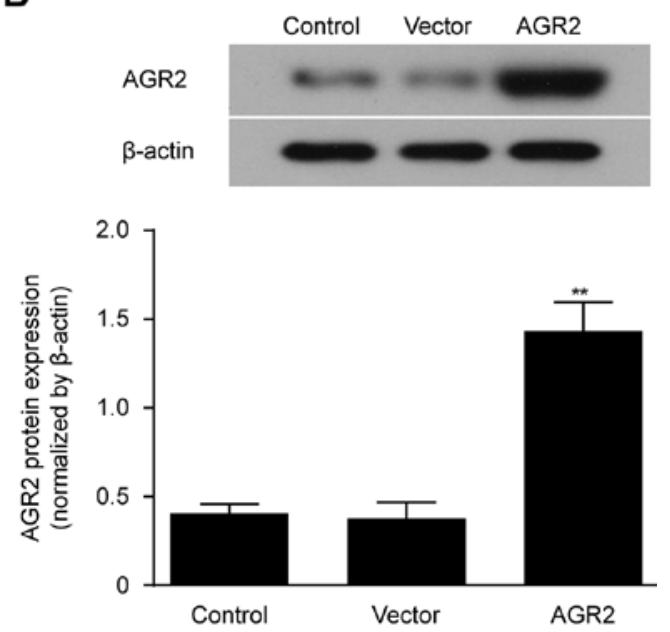

Figure 2. Both anterior gradient protein 2 homologue (AGR2) mRNA expression (A) and (B) protein expression were significantly higher in Caco-2 cells transfected with the AGR2 plasmids than in the control cells. The results are presented as the mean $\pm \mathrm{SD}$. ${ }^{* *} \mathrm{P}<0.01$ compared with the control group.

A

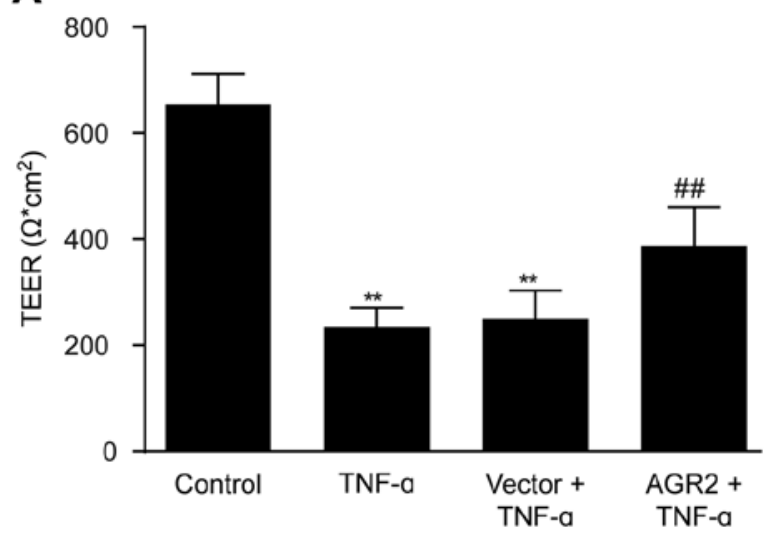

B

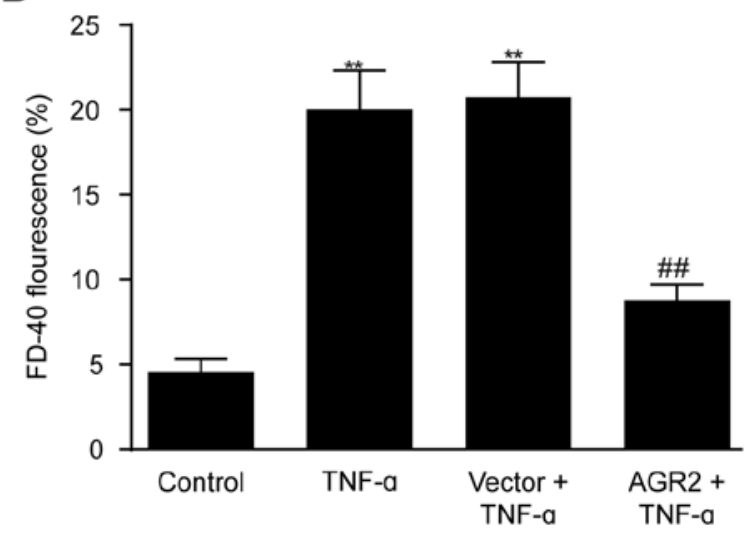

Figure 3. (A) Anterior gradient protein 2 homologue (AGR2) plasmid transfection significantly inhibited the decrease in transepithelial electrical resistance (TEER) induced by rhTNF- $\alpha$. (B) AGR2 plasmid transfection significantly inhibited the increase in fluorescein isothiocyanate (FITC)-dextran (40 kDa) flux (FD-40) induced by rhTNF- $\alpha$. The results are presented as the mean \pm SD. ${ }^{* *} \mathrm{P}<0.01$ compared with the control group; ${ }^{\# \#} \mathrm{P}<0.01 \mathrm{compared}$ with the TNF- $\alpha$ group.

occludin and ZO-1 appeared as a continuous band surrounding the cell boundary, with no obvious gap. After $48 \mathrm{~h}$ of incubation with TNF- $\alpha$, these proteins had rough edges and an interrupted distribution; there were visible irregular openings, with a honeycomb appearance between the cells of the monolayers. AGR2 plasmid transfection significantly reduced the TNF- $\alpha$ induced damage to the TJs, causing the surfaces of the proteins to become completely adherent (Fig. 4D and E). These results indicated that AGR2 inhibited the TNF- $\alpha$-induced decreases in expression of TJ proteins.

AGR2 inhibits TNF- $\alpha$-induced cytoskeletal $F$-actin rearrangement. Immunofluorescence staining showed that in the control group, F-actin was arranged in the cytoplasm in neat rows close to the cell membrane, forming a dense, smooth, continuous ring with a uniform distribution. After $48 \mathrm{~h}$ of incubation with TNF- $\alpha$, cytoskeletal F-actin was rearranged, forming a fuzzy, peripheral actin ribbon with a decrease in the cortical F-actin fibre macula densa. Some cells had obvious transcellular stress fibre formation. AGR2 plasmid transfection inhibited the rear- rangement of cytoskeletal F-actin, which appeared as a clear, peripheral actin ribbon with a decrease in cytoplasmic stress fibres (Fig. 5). The results indicated that AGR2 inhibited the TNF- $\alpha$-induced rearrangement of cytoskeletal F-actin.

AGR2 inhibits TNF- $\alpha$ activation of the $M L C K / p-M L C$ pathway. The western blotting results showed that the expression of MLCK and p-MLC was significantly higher in cells incubated with TNF- $\alpha$ than levels noted in the control cells. Transfection with the AGR2 gene plasmid inhibited the TNF- $\alpha$-induced elevations in MLCK and p-MLC expression (Fig. 6A and B). These results indicated that AGR2 attenuated the TNF- $\alpha$-induced intestinal mucosal barrier injury by regulating the MLCK/p-MLC pathway.

AGR2 reduces $T N F$ - $\alpha$-induced barrier damage by inhibiting activation of $N F-\kappa B$ p 65 . Western blotting showed that TNF- $\alpha$ significantly increased nuclear $\mathrm{NF}-\kappa \mathrm{B}$ p65 protein expression and decreased cytoplasmic NF- $\mathrm{B}$ p65 expression compared with that in the control group. In contrast, nuclear expression 
A
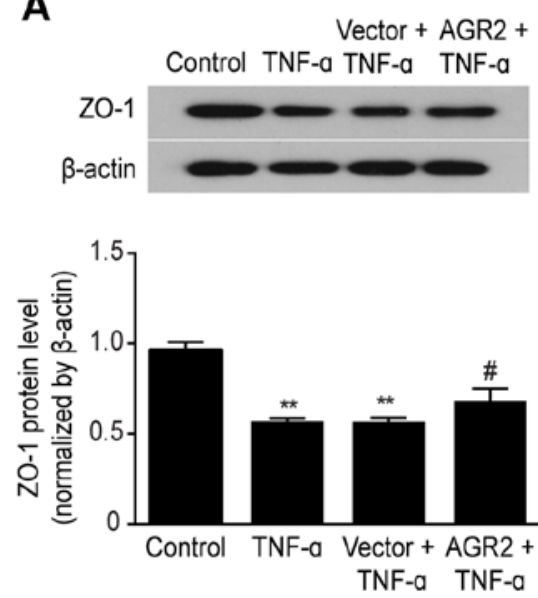

D
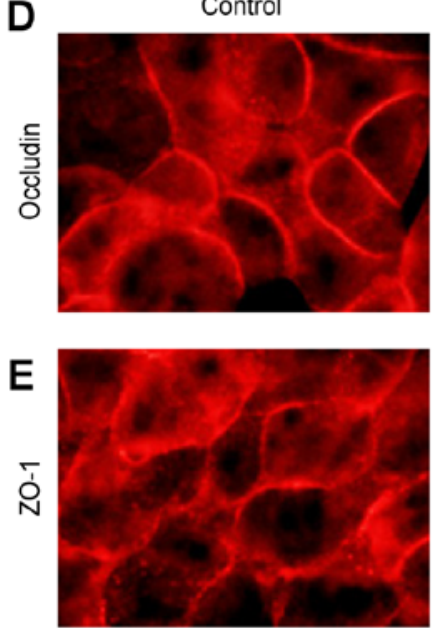

B
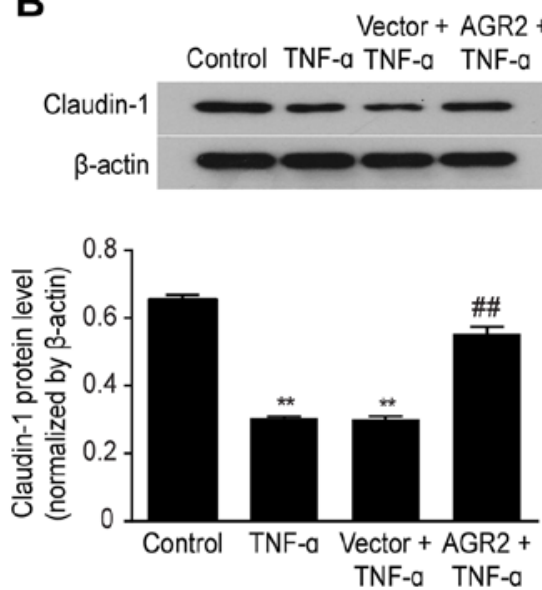

TNF-a
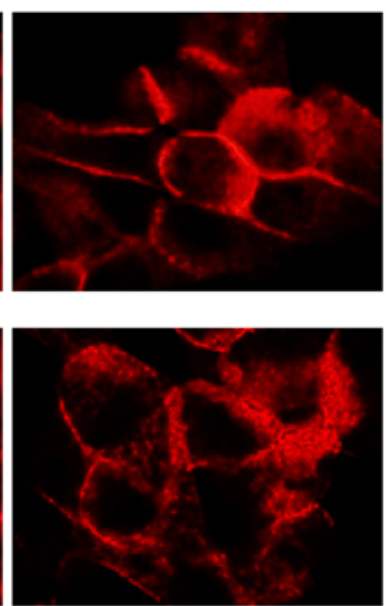

C
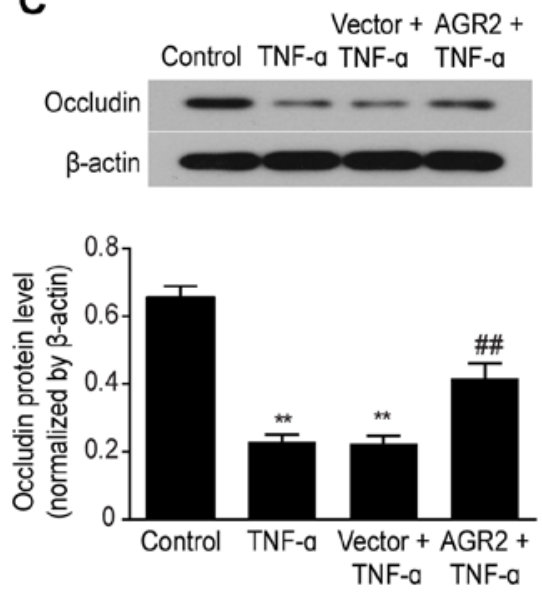

Figure 4. Western blot analysis showed that anterior gradient protein 2 homologue (AGR2) inhibited the decreased expression of tight junction (TJ) proteins (A) zonula occludens-1 (ZO-1), (B) claudin-1 and (C) occludin induced by TNF- $\alpha$. The results are presented as the mean \pm SD. ${ }^{*} \mathrm{P}<0.01$ compared with controls; ${ }^{*} \mathrm{P}<0.05$ compared with rhTNF- $\alpha$-treated cells; ${ }^{\# \#} \mathrm{P}<0.01$ compared with rhTNF- $\alpha$-treated cells. Immunofluorescence staining shows that AGR2 inhibited the rhTNF- $\alpha$-induced abnormal distribution of (D) occludin and (E) ZO-1 proteins.
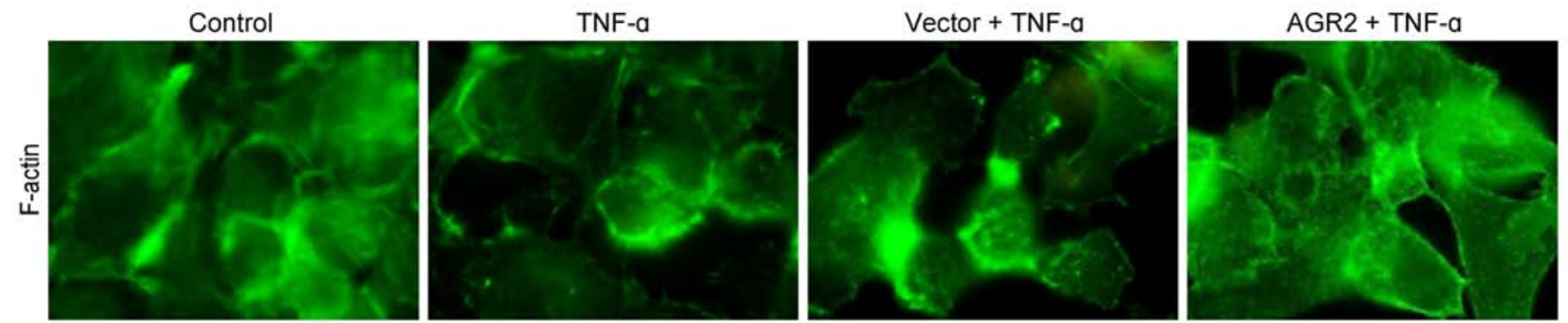

Figure 5. Immunofluorescence staining shows that anterior gradient protein 2 homologue (AGR2) inhibited the cytoskeletal F-actin rearrangement induced by TNF- $\alpha$ and stabilized the cytoskeletal structure.

of this protein was decreased and cytoplasmic expression was increased in the Caco-2 cells transfected with the AGR2 plasmids (Fig. 7A and B). Immunofluorescence staining further confirmed the above results, showing that in the control group, the NF- $\mathrm{KB}$ p 65 protein was mainly localized to the cytoplasm, where it was inactive. However, in the TNF- $\alpha$-stimulated Caco- 2 cells, this protein was predominantly nuclear. In the Caco-2 cells transfected with the AGR2 plasmids, nuclear accumulation of NF- $\kappa B$ p65 in response to TNF- $\alpha$ was signifi- cantly reduced (Fig. 7C). The results indicate that AGR2 may reduce intestinal barrier damage by inhibiting the activation of NF-kB p65.

\section{Discussion}

The TJ complex is critical for maintaining mucosal barrier integrity after injury (30); however, the exact mechanism for primary repair of TJs has not been fully elucidated. The 
A
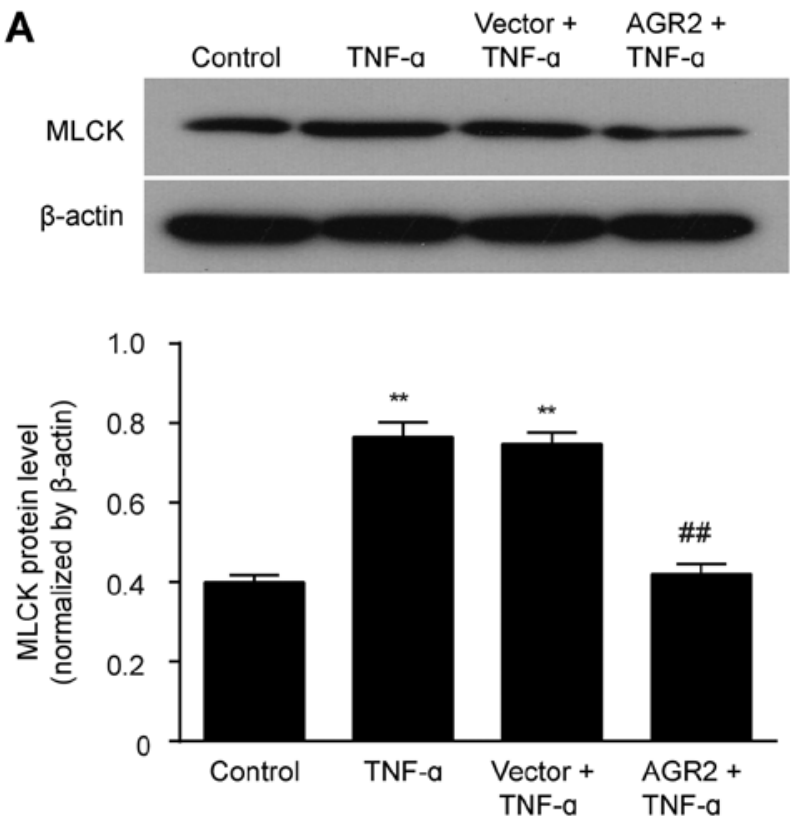

B
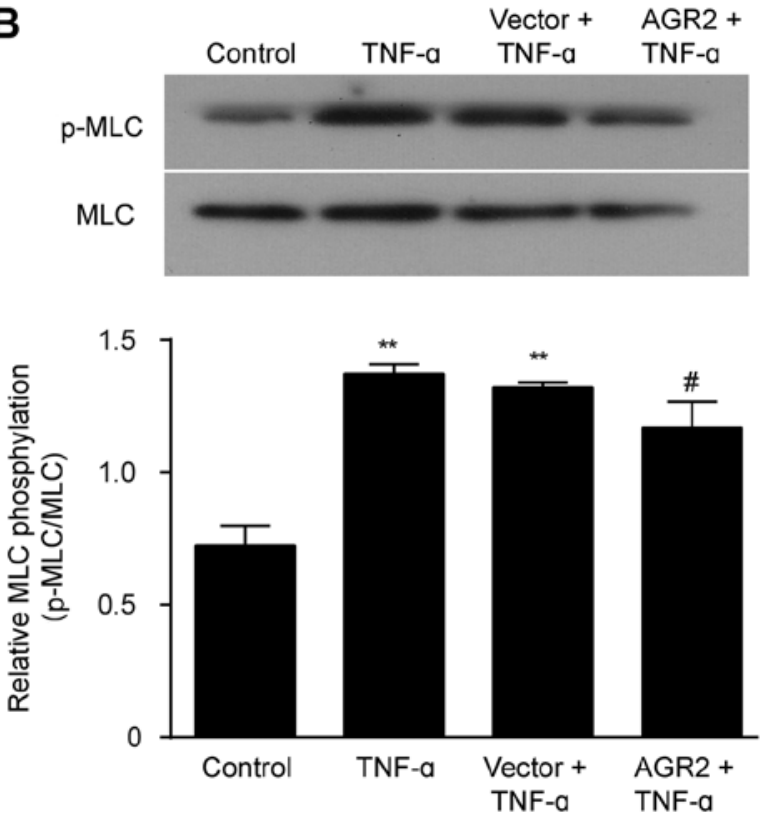

Figure 6. Western blot analysis showed that anterior gradient protein 2 homologue (AGR2) inhibited the rhTNF- $\alpha$-induced expression of (A) myosin light chain kinase (MLCK) and (B) p-MLC. The results are presented as the mean \pm SD. ${ }^{* *} \mathrm{P}<0.01$ compared with the controls; ${ }^{\sharp} \mathrm{P}<0.05$ compared with the rhTNF- $\alpha$ group; ${ }^{\#} \mathrm{P}<0.01$ compared with the TNF- $\alpha$ group.

present study describes a novel mechanism by which AGR2 regulates TJ proteins and the cytoskeleton in response to TNF- $\alpha$-induced intestinal barrier dysfunction. We report that TNF- $\alpha$ decreased AGR2 expression in the Caco- 2 monolayers, while AGR2 overexpression inhibited the TNF- $\alpha$-mediated intestinal barrier injury, reduced the permeability of Caco-2 monolayers, increased the expression of TJ proteins and stabilized the cytoskeletal structure by inhibiting NF- $\mathrm{B}$ p65 mediated MLCK/p-MLC pathway activation.

Numerous previous studies, including those by our group, have confirmed that inflammatory cytokine-mediated intestinal epithelial barrier dysfunction contributes to multiple enteropathies, including IBD (31-34); such cytokines disrupt the integrity of the TJ complex and increase intestinal barrier permeability. A study by Chen et al showed that stimulating Caco-2 monolayers with $10 \mathrm{ng} / \mathrm{ml}$ TNF- $\alpha$ did not affect $\mathrm{TJ}$ protein expression levels but that $\mathrm{TNF}-\alpha$ played a role in altering the distribution of $\mathrm{TJ}$ proteins (35). Other studies revealed that $10 \mathrm{ng} / \mathrm{ml} \mathrm{TNF-} \alpha$ led to both decrease expression and abnormal distribution of TJ proteins $(36,37)$. In our study, stimulating Caco-2 monolayers with $100 \mathrm{ng} / \mathrm{ml} \mathrm{TNF-} \alpha$ significantly increased cell membrane permeability, accompanied by abnormal distribution and decreases in the expression of ZO-1, claudin-1 and occludin, as well as rearrangement of cytoskeletal F-actin; these findings are consistent with a study by Zhang et al (38). Our data further strengthens the evidence that TNF- $\alpha$ can disrupt the intestinal epithelial barrier by reducing TJ protein levels and reorganizing cytoskeletal structure.

More recently, it has been demonstrated that activation of the MLCK/p-MLC pathway plays a key role in inflammatory cytokine-mediated intestinal mucosal barrier dysfunction $(39,40)$. To investigate the molecular mechanism by which TNF- $\alpha$ injures the intestinal barrier, we determined MLCK, MLC and p-MLC protein expression and found that TNF- $\alpha$-induced barrier injury was accompanied by increases in both MLCK expression and MLC phosphorylation, which was also consistent with the findings of Cunningham et al (41).

Additionally, activation of NF- $\kappa \mathrm{B}$ p 65 also plays a crucial role in TNF- $\alpha$-induced intestinal barrier injury. Previous research has shown that NF- $\kappa \mathrm{B}$ binds to the promoter region of the $M L C K$ gene and increases MLCK transcription and MLC phosphorylation, leading to the altered permeability and TJ disruption known to be associated with proinflammatory cytokines (42). Here, in our study, TNF- $\alpha$ significantly increased nuclear protein expression and distribution of NF- $\mathrm{NB}$ p 65 , indicating that $\mathrm{NF}-\kappa \mathrm{B}$ p 65 is related to the intestinal barrier injury caused by TNF- $\alpha$, consistent with the findings of Al-Sadi et al (43).

AGR2 is a recently discovered gene with a protective effect of mucosal barrier in IBD; however, the mechanism by which it regulates intestinal epithelial barrier function remains unclear. In the present study, we investigated the role of AGR2 in TNF- $\alpha$-induced intestinal barrier injury and its related mechanisms. The results demonstrated that overexpression of AGR2 inhibited the TNF- $\alpha$-induced decrease in TEER and increase in FD-40 flux, increased the expression and improved the distribution of TJ proteins, and stabilized the cytoskeletal structure, thereby ameliorating the TNF- $\alpha$-induced cell membrane hyperpermeability. The impact of AGR2 on TNF- $\alpha-$ induced activation of the MLCK/p-MLC signaling pathway was then detected; AGR2 inhibited the changes in MLCK, MLC and p-MLC expression in response to TNF- $\alpha$ stimulation, preliminarily confirming that AGR2 improved intestinal barrier dysfunction through regulation of the MLCK/p-MLC pathway. Subsequently, we investigated whether activity of the transcription factor NF- $\kappa \mathrm{B}$ p 65 was also influenced by AGR2. The results revealed that AGR2 inhibited nulear protein expression of $\mathrm{NF}-\kappa \mathrm{B}$ p65, and immunofluorescence staining revealed that AGR2 inhibited NF- $\kappa \mathrm{B}$ p65 translocation into the nucleus. Thus, these data suggest that inhibition 
A
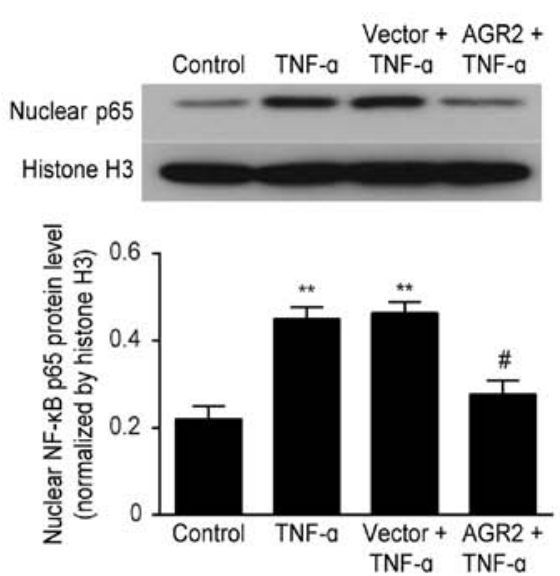

B
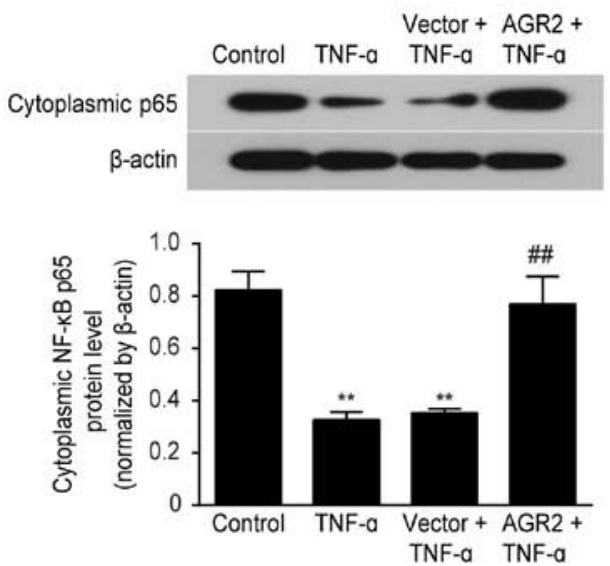

\section{C}
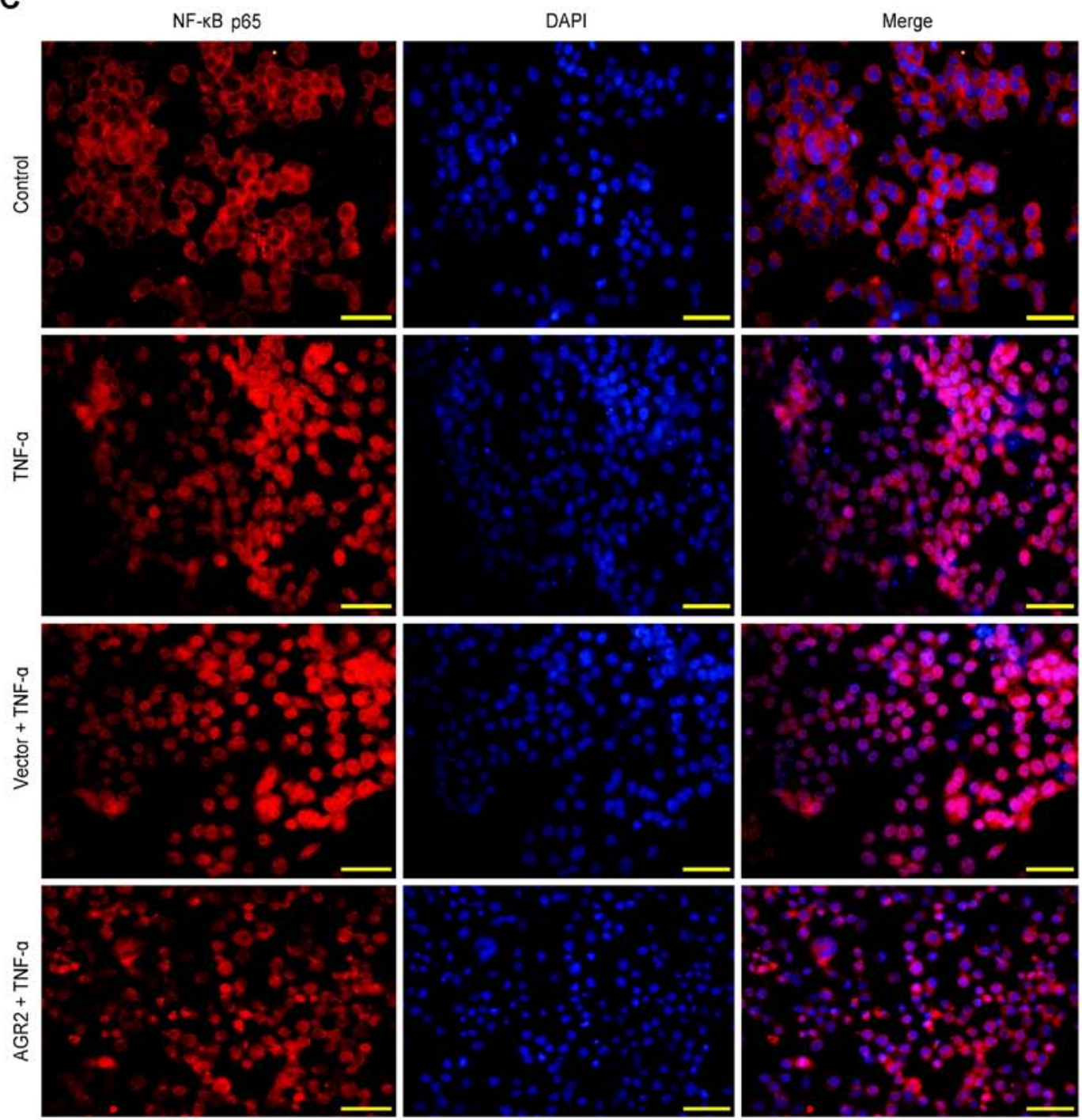

Figure 7. Western blot analysis showed that anterior gradient protein 2 homologue (AGR2) inhibited the rhTNF- $\alpha$-induced increase in (A) nuclear NF- $\kappa B$ p65 expression and decrease in (B) cytoplasmic NF- $\mathrm{kB}$ p 65 expression. The results are presented as the mean $\pm \mathrm{SD}$. ${ }^{* *} \mathrm{P}<0.01$ compared with controls; ${ }^{* \#} \mathrm{P} 0.01$ compared with the rhTNF- $\alpha$ group. (C) Immunofluorescence staining showed that AGR2 inhibited the nuclear translocation of NF- $\kappa \mathrm{B}$ p65 induced by rhTNF- $\alpha$.

of NF- $\kappa \mathrm{B}$ p65-mediated MLCK/p-MLC pathway activation may be the molecular mechanism by which AGR2 ameliorates intestinal barrier dysfunction caused by TNF- $\alpha$.

One limitation of the present study is that we tested the effect of AGR2 on NF- $\mathrm{kB}$ p65 and MLCK/p-MLC pathway expression in TNF- $\alpha$-induced intestinal epithelial barrier dysfunction, but we did not specific block NF-kB p65 or MLCK gene expression. Further study is needed to investigate other potential mechanisms by which AGR2 ameliorates TNF- $\alpha$-induced intestinal epithelial barrier injury. 
In conclusion, to the best of our knowledge, this is the first study showing that the $A G R 2$ gene inhibits TNF- $\alpha$-induced intestinal epithelial hyperpermeability by enhancing TJ function and cytoskeletal stability. These effects may be exerted through the inhibition of NF- $\kappa \mathrm{B}$ and MLCK/p-MLC pathway activation. In addition, this study may provide new evidence for treating IBD by upregulating AGR2 expression, and we hope that this study potentially helps improve the prognosis of IBD patients.

\section{Acknowledgements}

The authors are grateful to the members of the Department of Pediatric Gastroenterology, China Medical University. We would also like to thank Ms. Dongyan Liu for assisting with the manuscript revision.

\section{References}

1. Salim SY and Söderholm JD: Importance of disrupted intestinal barrier in inflammatory bowel diseases. Inflamm Bowel Dis 17: 362-381, 2011.

2. Petersson J, Schreiber O, Hansson GC, Gendler SJ, Velcich A, Lundberg JO, Roos S, Holm L and Phillipson M: Importance and regulation of the colonic mucus barrier in a mouse model of colitis. Am J Physiol Gastrointest Liver Physiol 300: G327-G333, 2011.

3. Fernández-Blanco JA, Estévez J, Shea-Donohue T, Martínez V, and Vergara P: Changes in epithelial barrier function in response to parasitic infection: implications for IBD pathogenesis. J Crohns Colitis 9: 463-476, 2015.

4. Edelblum KL and Turner JR: The tight junction in inflammatory disease: communication breakdown. Curr Opin Pharmacol 9: 715-720, 2009.

5. Alipour M, Zaidi D, Valcheva R, Jovel J, Martínez I, Sergi C, Walter J, Mason AL, Wong GK, Dieleman LA, et al: Mucosal barrier depletion and loss of bacterial diversity are primary abnormalities in paediatric ulcerative colitis. J Crohns Colitis 10: 462-471, 2016.

6. Vetrano S, Rescigno M, Cera MR, Correale C, Rumio C, Doni A, Fantini M, Sturm A, Borroni E, Repici A, et al: Unique role of junctional adhesion molecule-A in maintaining mucosal homeostasis in inflammatory bowel disease. Gastroenterology 135 : $173-184,2008$

7. Neurath MF and Travis SP: Mucosal healing in inflammatory bowel diseases: a systematic review. Gut 61: 1619-1635, 2012.

8. Suzuki T, Yoshinaga N and Tanabe S: Interleukin-6 (IL-6) regulates claudin-2 expression and tight junction permeability in intestinal epithelium. J Biol Chem 286: 31263-31271, 2011

9. Amasheh M, Grotjohann I, Amasheh S, Fromm A, Söderholm JD, Zeitz M, Fromm M and Schulzke JD: Regulation of mucosal structure and barrier function in rat colon exposed to tumor necrosis factor alpha and interferon gamma in vitro: a novel model for studying the pathomechanisms of inflammatory bowel disease cytokines. Scand J Gastroenterol 44: 1226-1235, 2009.

10. Liu H, Wang P, Cao M, Li M and Wang F: Protective role of oligomycin against intestinal epithelial barrier dysfunction caused by IFN- $\gamma$ and TNF- $\alpha$. Cell Physiol Biochem 29: 799-808, 2012.

11. Michielan A and D'Incà R: Intestinal permeability in inflammatory bowel disease: pathogenesis, clinical evaluation, and therapy of leaky gut. Mediators Inflamm 2015: 628157, 2015.

12. Suzuki T: Regulation of intestinal epithelial permeability by tight junctions. Cell Mol Life Sci 70: 631-659, 2013.

13. Shen L, Weber CR, Raleigh DR, Yu D and Turner JR: Tight junction pore and leak pathways: a dynamic duo. Annu Rev Physiol 73: 283-309, 2011

14. Chen Y, Li D, Dai Z, Piao X, Wu Z, Wang B, Zhu Y and Zeng Z: L-methionine supplementation maintains the integrity and barrier function of the small-intestinal mucosa in post-weaning piglets. Amino Acids 46: 1131-1142, 2014.

15. Turner JR: Intestinal mucosal barrier function in health and disease. Nat Rev Immunol 9: 799-809, 2009.

16. Marchiando AM, Shen L, Graham WV, Edelblum KL, Duckworth CA, Guan Y, Montrose MH, Turner JR and Watson AJ: The epithelial barrier is maintained by in vivo tight junction expansion during pathologic intestinal epithelial shedding. Gastroenterology 140: 1208-1218, 2011.
17. Assasi N, Blackhouse G, Xie F, Marshall JK, Irvine EJ, Gaebel K, Robertson D, Campbell K, Hopkins R and Goeree R: Patient outcomes after anti TNF-alpha drugs for Crohn's disease. Expert Rev Pharmacoecon Outcomes Res 10: 163-175, 2010.

18. Wang QY, Sun AM, Song J, Chen Y, Wang JD and Li CG: Cytokine tumor necrosis factor alpha induces intestinal epithelial barrier dysfunction. Cytokine 58: 226-230, 2012.

19. Huys L, Van Hauwermeiren F, Dejager L, Dejonckheere E, Lienenklaus S, Weiss S, Leclercq G and Libert C: Type I interferon drives tumor necrosis factor-induced lethal shock. J Exp Med 206: 1873-1882, 2009.

20. Takahashi N, Vanlaere I, de Rycke R, Cauwels A, Joosten LA, Lubberts E, van den Berg WB and Libert C: IL-17 produced by Paneth cells drives TNF-induced shock. J Exp Med 205: 1755-1761, 2008.

21. Suzuki M, Nagaishi T, Yamazaki M, Onizawa M, Watabe T, Sakamaki Y, Ichinose S, Totsuka M, Oshima S, Okamoto R, et al: Myosin light chain kinase expression induced via tumor necrosis factor receptor 2 signaling in the epithelial cells regulates the development of colitis-associated carcinogenesis. PLoS One 9: e88369, 2014

22. Feng Y and Teitelbaum DH: Tumour necrosis factor-induced loss of intestinal barrier function requires TNFR1 and TNFR2 signalling in a mouse model of total parenteral nutrition. J Physiol 591: 3709-3723, 2013.

23. Du J, Chen Y, Shi Y, Liu T, Cao Y, Tang Y, Ge X, Nie H, Zheng $\mathrm{C}$ and Li YC: 1,25-Dihydroxyvitamin D protects intestinal epithelial barrier by regulating the myosin light chain kinase signaling pathway. Inflamm Bowel Dis 21: 2495-2506, 2015.

24. Liu X, Xu J, Mei Q, Han L and Huang J: Myosin light chain kinase inhibitor inhibits dextran sulfate sodium-induced colitis in mice. Dig Dis Sci 58: 107-114, 2013.

25. Park SW, Zhen G, Verhaeghe C, Nakagami Y, Nguyenvu LT, Barczak AJ, Killeen N and Erle DJ: The protein disulfide isomerase AGR2 is essential for production of intestinal mucus. Proc Natl Acad Sci USA 106: 6950-6955, 2009.

26. Zheng W, Rosenstiel P, Huse K, Sina C, Valentonyte R, Mah N, Zeitlmann L, Grosse J, Ruf N, Nürnberg P, et al: Evaluation of $A G R 2$ and $A G R 3$ as candidate genes for inflammatory bowel disease. Genes Immun 7: 11-18, 2006.

27. Lai YR, Lu YF, Lien HW, Huang CJ and Hwang SP: Foxa2 and Hiflab regulate maturation of intestinal goblet cells by modulating agr2 expression in zebrafish embryos. Biochem $\mathrm{J}$ 473: 2205-2218, 2016.

28. Zhao F, Edwards R, Dizon D, Afrasiabi K, Mastroianni JR, Geyfman M, Ouellette AJ, Andersen B and Lipkin SM: Disruption of Paneth and goblet cell homeostasis and increased endoplasmic reticulum stress in Agr2 $2^{-/}$mice. Dev Biol 338: 270-279, 2010

29. Su L, Shen L, Clayburgh DR, Nalle SC, Sullivan EA, Meddings JB, Abraham C and Turner JR: Targeted epithelial tight junction dysfunction causes immune activation and contributes to development of experimental colitis. Gastroenterology 136 : 551-563, 2009

30. Liu Z, Li N and Neu J: Tight junctions, leaky intestines, and pediatric diseases. Acta Paediatr 94: 386-393, 2005.

31. Lin N, Xu LF and Sun M: The protective effect of trefoil factor 3 on the intestinal tight junction barrier is mediated by toll-like receptor 2 via a PI3K/Akt dependent mechanism. Biochem Biophys Res Commun 440: 143-149, 2013.

32. Xu LF, Teng X, Guo J and Sun M: Protective effect of intestinal trefoil factor on injury of intestinal epithelial tight junction induced by platelet activating factor. Inflammation 35 : 308-315, 2012.

33. Blander JM: Death in the intestinal epithelium-basic biology and implications for inflammatory bowel disease. FEBS J 283: 2720-2730, 2016

34. Xiao YT, Yan WH, Cao Y, Yan JK and Cai W: Neutralization of IL- 6 and TNF- $\alpha$ ameliorates intestinal permeability in DSS-induced colitis. Cytokine 83: 189-192, 2016.

35. Chen S, Zhu J, Chen G, Zuo S, Zhang J, Chen Z, Wang X, Li J, Liu Y and Wang P: 1,25-Dihydroxyvitamin $\mathrm{D}_{3}$ preserves intestinal epithelial barrier function from TNF-alpha induced injury via suppression of NF-kB p65 mediated MLCK-P-MLC signaling pathway. Biochem Biophys Res Commun 460: 873-878, 2015

36. He F, Peng J, Deng XL, Yang LF, Camara AD, Omran A, Wang GL, Wu LW, Zhang CL and Yin F: Mechanisms of tumor necrosis factor-alpha-induced leaks in intestine epithelial barrier. Cytokine 59: 264-272, 2012. 
37. Cao M, Wang P, Sun C, He W and Wang F: Amelioration of IFN- $\gamma$ and TNF- $\alpha$-induced intestinal epithelial barrier dysfunction by berberine via suppression of MLCK-MLC phosphorylation signaling pathway. PLoS One 8: e61944, 2013.

38. Zhang J, Lu Y, Wei J, Li L and Han L: Protective effect of carboxytmethylpachymaran on TNF-alpha-induced damage in Caco-2 cell monolayers. Int J Biol Macromol 93 (Pt A): 506-511, 2016.

39. Kuhlmann CR, Tamaki R, Gamerdinger M, Lessmann V, Behl C, Kempski OS and Luhmann HJ: Inhibition of the myosin light chain kinase prevents hypoxia-induced blood-brain barrier disruption. J Neurochem 102: 501-507, 2007.

40. Boivin MA, Ye D, Kennedy JC, Al-Sadi R, Shepela C and Ma TY: Mechanism of glucocorticoid regulation of the intestinal tight junction barrier. Am J Physiol Gastrointest Liver Physiol 292: G590-G598, 2007.
41. Cunningham KE and Turner JR: Myosin light chain kinase: pulling the strings of epithelial tight junction function. Ann NY Acad Sci 1258: 34-42, 2012.

42. Ye D and Ma TY: Cellular and molecular mechanisms that mediate basal and tumour necrosis factor-alpha-induced regulation of myosin light chain kinase gene activity. J Cell Mol Med 12: 1331-1346, 2008.

43. Al-Sadi R, Guo S, Ye D, Rawat M and Ma TY: TNF-alpha modulation of intestinal tight junction permeability is mediated by NIK/IKK-alpha axis activation of the canonical NF- $\kappa$ B pathway. Am J Pathol 186: 1151-1165, 2016. 